\title{
Digital Data, Virtual Tours, and 3D Models Integration Using an Open-Source Platform
}

\author{
Nicola Maiellaro ${ }^{(\bowtie)}$, Antonietta Varasano, and Salvatore Capotorto \\ Construction Technologies Institute, National Research Council of Italy, \\ via Lembo 38/B, 70124 Bari, Italy \\ \{maiellaro, varasano, capotorto\}@itc.cnr.it
}

\begin{abstract}
The site of the 'Balsignano village' in Modugno (Italy), for some years has been the object of attention by scholars. Recently renovated, its valuation is nowadays the main objective for that Municipality. The aim of this article is describing our approach in integrating digital data, virtual tours and $3 \mathrm{~d}$ models of the village elements to produce an interactive tool available on the internet, stimulating the desire to physically visit the settings. Our findings can be generalized to any context in which a link between a website and a physical site represent a challenge for low-budgeted Administrations.
\end{abstract}

Keywords: Virtual tour $\cdot$ Omni-directional cameras $\cdot 3 \mathrm{D}$ models Multi-camera systems $\cdot$ Joomla

\section{Introduction}

The spatial documentation of cultural heritage sites using conventional surveying methods based on laser scanning and photogrammetry has been well established for conservation and restoration - see, for example, the summary of metric survey techniques for heritage documentation in [1]. Digital technologies have increased speed and completeness in that kind of survey, offering new opportunities to collect, analyzing and disseminate information [2], according to a project's scope timeline and budget constraints. However, due to the high costs of laser scanners, two approaches have been suggested for an intermediate documentation typology: the use of Virtual tours combined to Information Modelling [3] and the extraction of 3D structure from images captured from multiple viewpoints, utilizing different type of camera [4]. Moreover, the main focus of recent research is the visual, geometric, and textural characteristics of a single monument, while integration with other monuments and additional information - such as historical overview, detailed description, and location-are missing [5]. For example, a study on an exhaustive sample of 148 archaeology websites [6] shows that the $80 \%$ provides contextual information, $50 \%$ share data textually, using simple html pages or link to PDF document and only one website has virtual data in the form of an interactive map with data and image visualized in a callout [7]. In this article, we illustrate digital data, virtual tours and $3 \mathrm{~d}$ models for representing selected objects located in 'Balsignano Village', integrated in a website with an intuitive interface (Fig. 1). 


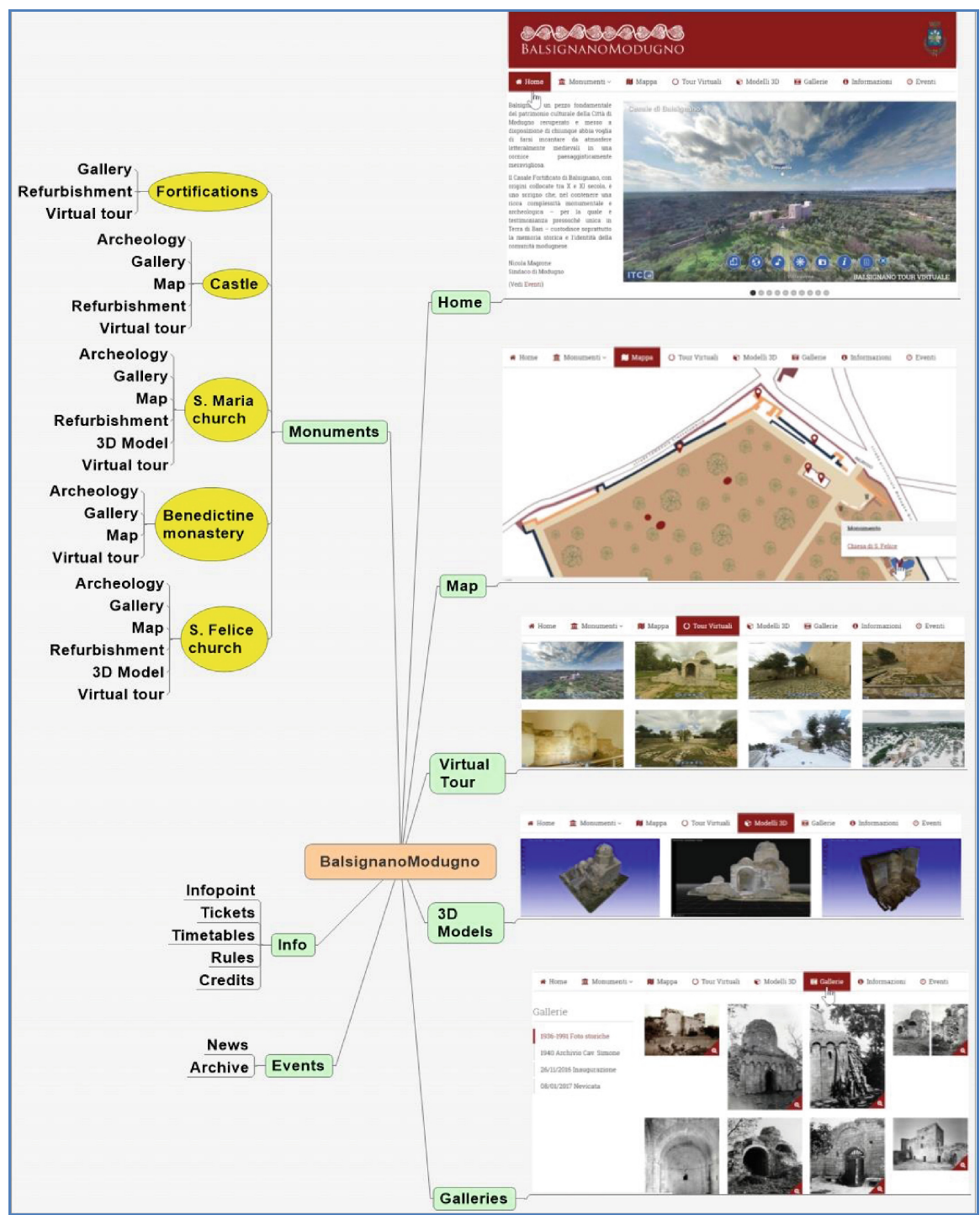

Fig. 1. The logical scheme of the website [8]: menu items (green background), Point of Interest (yellow background), and their contents (transparent background). (Color figure online)

\section{The Case Study}

The village, which is near a branch of the 'Via Traiana', owes much of its charm to the suggestive position in a context that has still preserved the natural appearance of the Apulian agricultural landscape, characterized by 'trulli', rock settlements, dry stone walls, areas for grapes pressing, natural ice storages. 
The first historical documentation on Balsignano consists of one parchment of May 962, preserved at the Basilica of 'S. Nicola' of Bari. Balsignano develops between the 10th and the 11th centuries as a fortified settlement, served by local roads.

Destroyed a first time in 988 following a Saracen raid, it was rebuilt and donated in 1092 by the Duke Norman Ruggero to the distant 'S. Lorenzo' Benedictine abbey of Aversa. Finally, Balsignano was devastated in the sixteenth century by French and Spanish troops who contended for hegemony in southern Italy [9]. Currently there are:

- a substantial part of Fortifications, with slit openings;

- the Castle (Fig. 2);

- 'S. Maria' church (14th century);

- the Benedictine monastery;

- 'S. Felice' church (11th century).

In 1981, Balsignano, as exceptional example of a medieval complex, was subjected to the protection provided for by the Italian law 1089/39. The works carried out over the last twenty-five years, due to the constant lack of funds, have concentrated on the structural and functional recovery of individual buildings. The complex is opened to the public since November 2016.

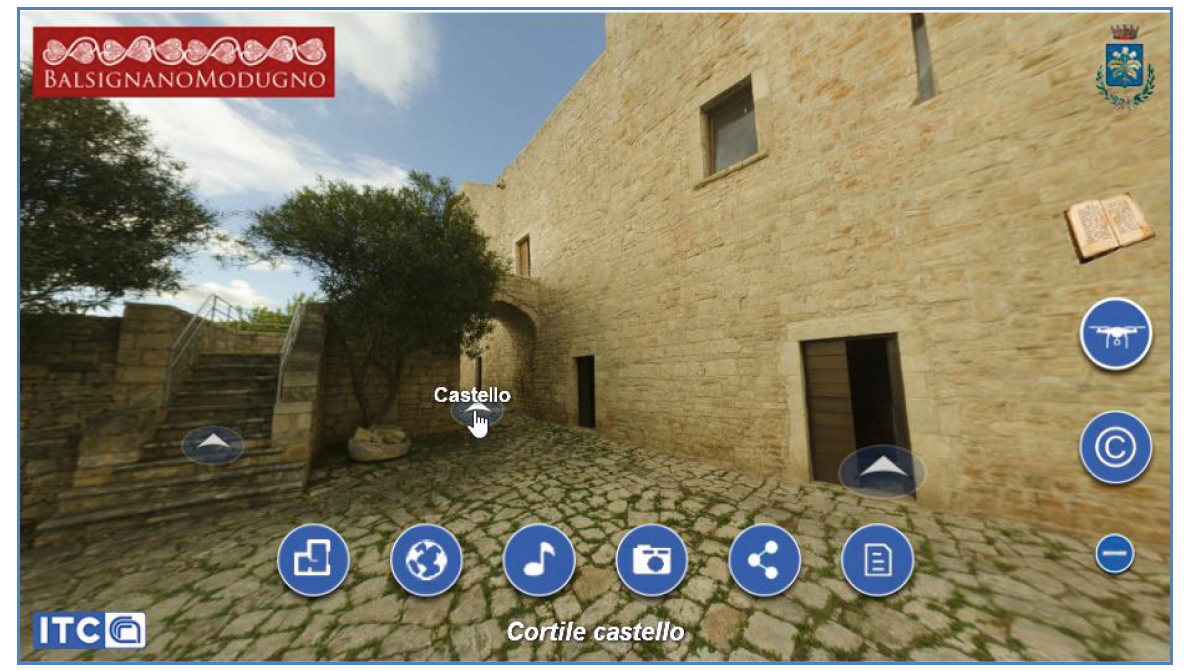

Fig. 2. Virtual tour of the Castle - external side.

\section{The Website Contents}

The objectives pursued in the design and implementation of the website are:

- allowing users to explore the contents of the archive through the integration of this material into virtual tours and 3D models 
- enhancing the sense of cultural presence, which can help to achieve the cultural aims of the project;

- ensuring accessibility and wider dissemination of the project.

In the following, we will describe the three main stages characterizing the development of any application [10]:

- documentation (gathering of information);

- representation (technical aspects of the digitization);

- dissemination (technical aspect about information and knowledge presentation).

\subsection{Documentation}

The primary source of documentation for the project were books $[11,12]$ and the photo and graph archives ('Galleries' in Fig. 1) of the Archaeological Superintendence of Puglia Region, the Architectural and Landscape Heritage Superintendence for Bari, BAT and Foggia, and the 'Simone' section [13] in the Picture Gallery 'Corrado Giaquinto' in Bari. Other valuable information was found on the Internet [9].

This significant amount of documents was selected to establish accurate references between sources and descriptions available in the representation output.

\subsection{Representation}

One of the factors considered in our work was to offer a visual feedback, improving the sense of presence. Thus, we followed two approaches, both image based, to produce virtual tours and $3 \mathrm{~d}$ models.

Virtual Tours. Computer-generated representation of the real world are modeled through the personal perception of reality and are time-consuming using traditional Virtual Reality techniques. On the contrary, virtual tours are unbroken view of a whole scene surrounding an observer, giving a sense of presence [14].

The most common systems include a network of predefined viewing locations linked together to create various path.

They use a sphere projection in 'equirectangular' image having 2:1 width-high ratio, called 360-degree panorama.

There are many systems for acquisition of image suitable for building panoramas [15]. The most common systems include rotational single camera system: the panorama is created by stitching together several images (partially overlapping with each other for alignment purposes) taken by a rotating camera placed in an arbitrary viewpoint.

To hold up image quality at zooming, it is required to produce a high-resolution panorama, obtainable stitching images acquired through a telephoto lens equipped camera mounted on a rotating motorized mechanism.

The disadvantage of this concept is the long acquisition time and the need to edit images to equalize different exposures - especially outdoor-and delete moving objects, as walking people or moving cars.

To overcome these problems, we tested a multi-camera system made by assembling six fixed cameras in a prearranged angles pack specifically designed to capture the 
360-degree scene (Fig. 3, left), encountering some problems with their assembling and synchronization; moreover it has a high cost to provide high-quality imagery.
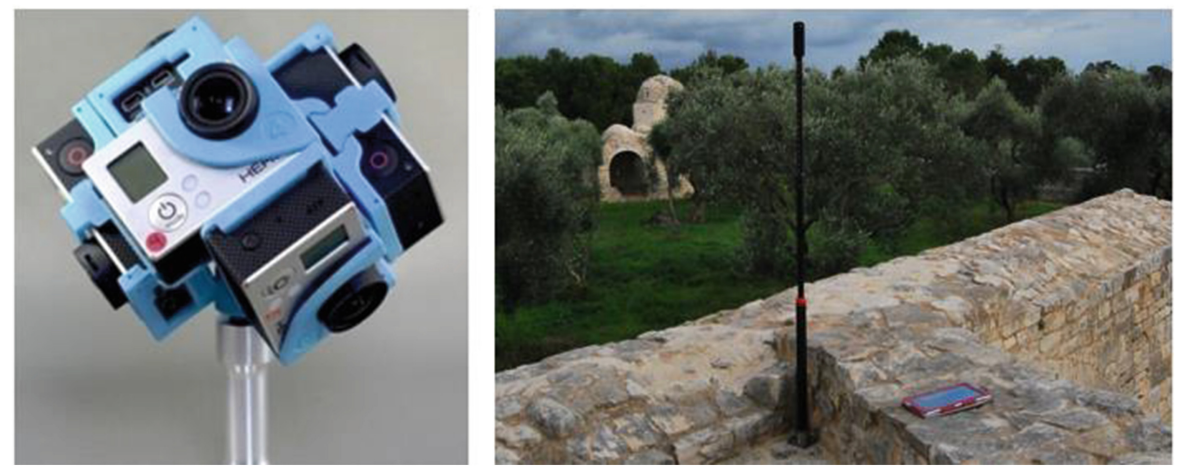

Fig. 3. 360-degree systems: Commercial six camera spherical array specifically designed (leftsource: Aerial Technology International, Oregon City, United States); Ricoh Teta Plus mounted on a telescopic rod in carbon fiber, with a cylindrical, low-radius, heavy base specifically designed to reduce its footprint (right).

An ultimate test was made using omni-directional cameras, having two back-toback sensors and fish-eye lenses: Nikon Key Mission and Ricoh Teta Plus (Fig. 3, right), producing respectively $7744 \times 3872$ and $5376 \times 2688$ pixel panoramas.

Although the Nikon camera is newer, the quality of the panorama produced by the stitching firmware is lower than that of the Ricoh camera (Fig. 4).
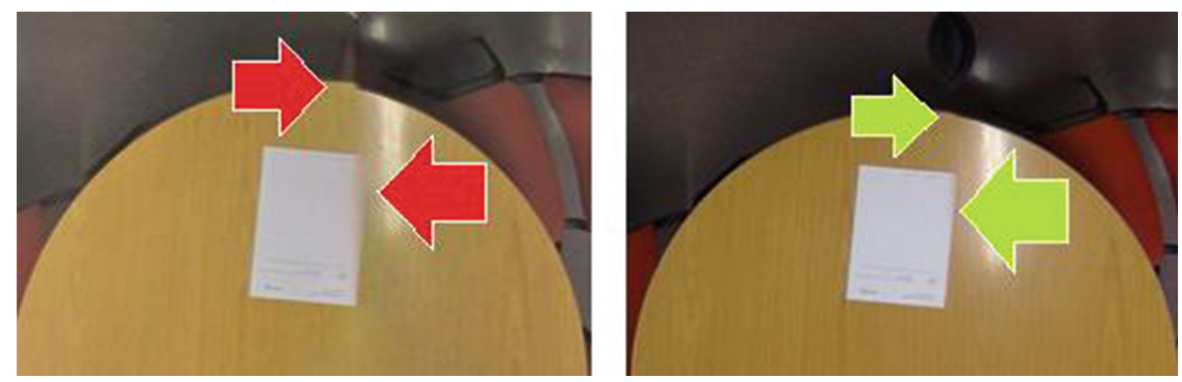

Fig. 4. Detail of panorama in the stitched area-laboratory test using two compact camera having two back-to-back sensors and fish-eye lenses. The results are poor for Nikon Key Mission camera (left) and good for the Ricoh Teta Plus camera (right)

We used the second one to shoot external panoramas instantly as that during a snowfall (Fig. 5), linked to a panorama produced using several shots from a camera mounted on a drone, to produce a virtual tour (Fig. 6). 


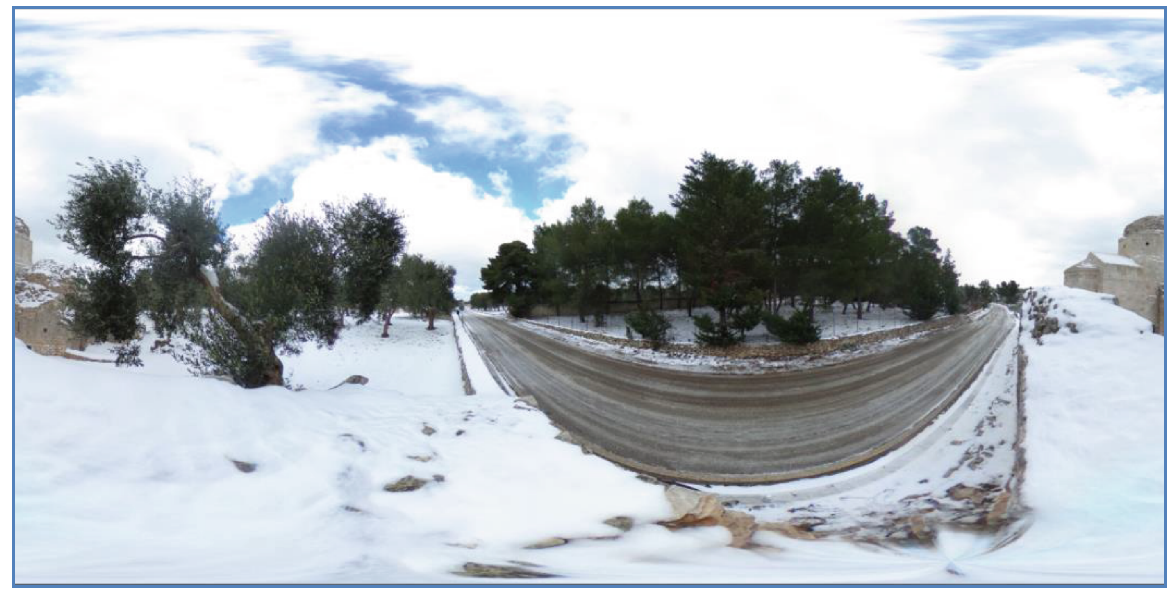

Fig. 5. Panorama - firmware stitched — by Ricoh Teta Plus, during a snowfall.
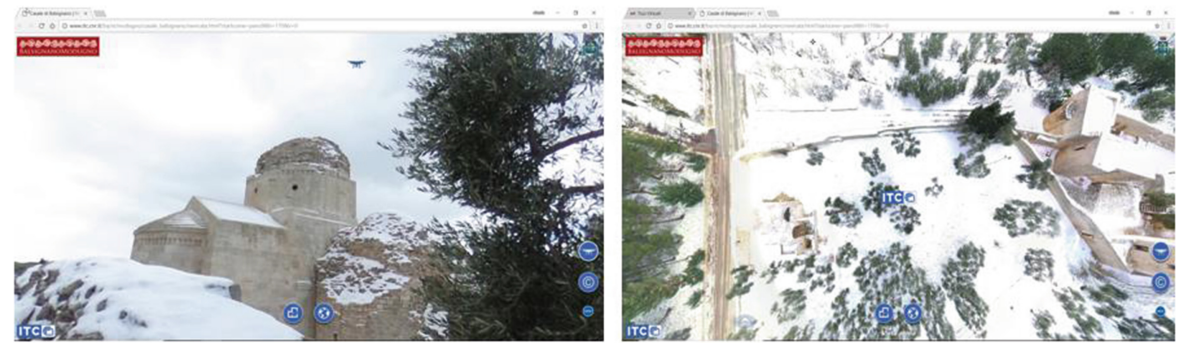

Fig. 6. Virtual tour during a snowfall, based on two panoramas linked through hotspots in the floorplan or clicking on the 'drone' button: terrestrial view (left) based on panorama in Fig. 5; aerial view by drone (right).

Finally, we utilized for indoor panoramas a wide-angle lens equipped cameramounted on a panoramic head - producing medium-resolution panoramas $(10.000 \times$ 5.000 pixel).

Panoramas are displayed on an interactive viewer, allowing their rotation, tilt, and zoom; most of them are just rotating images without further functionalities.

In the simplest application scenario, several individual panoramas are linked to each other using navigational hotspots to enter and exit scenes: when it is clicked in the panorama, the viewer is brought to the next panorama of the virtual tour.

To this end, we are now using AutoGarrow, a plug-in that allows the virtual tour navigation using the GPS coordinates of the camera generating panoramas. 
To improve the significance of the virtual tour, the panoramas developed have been enhanced using the Kolor Panotour Pro software, embedding - among others-hotspots to access different types of digital media (Fig. 7) as well as functional keys (blue circle with icon and white perimeter to stand out on dark backgrounds) to open:

- the floorplan with the location of the user and navigational hotspot;

- a google map with navigational hotspot of the whole project;

- a window with project info;

- a window with an image gallery.

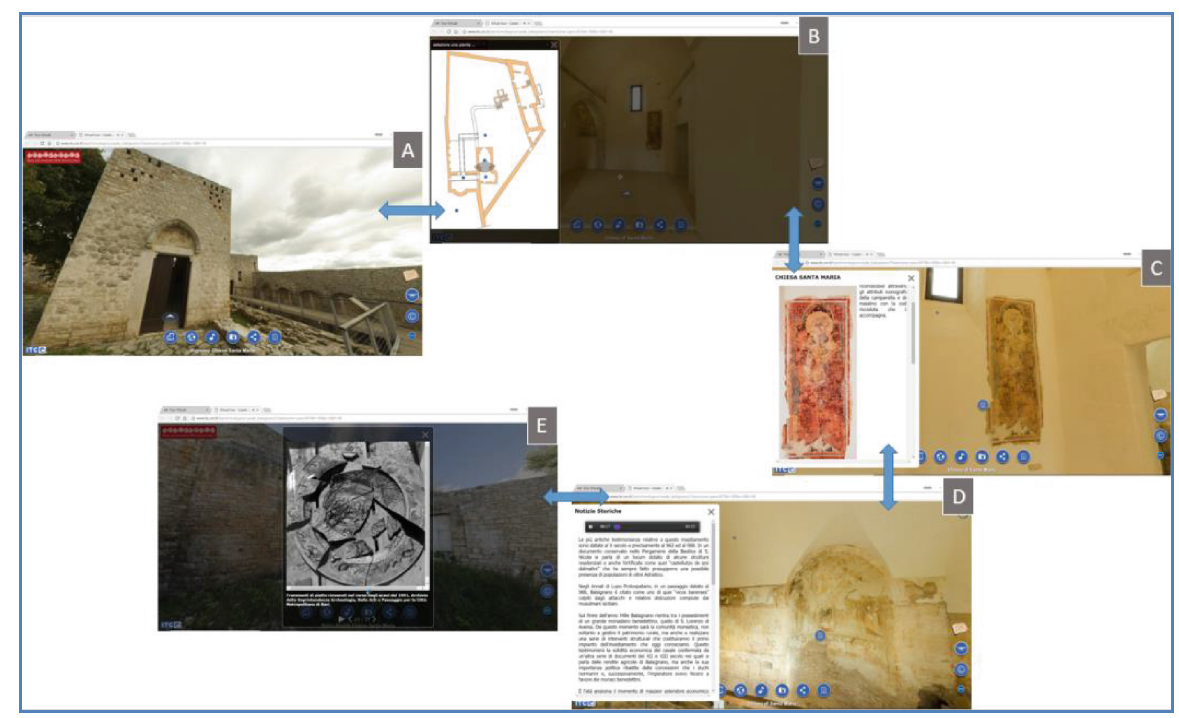

Fig. 7. Virtual tour of 'S. Maria' church organized into five linked panoramas: A. Main Entrance; B. Front nave (window on left: floorplan with hotspot); C. Back nave (window on left: info about the fresco); D. Lateral room (window on left: general historical info-text and sound); E. Rear outside view (window on center: historical image gallery).

3D Models. To navigate the virtual tour, the user can move between predefined viewing locations, which are linked together to create various paths as we have seen. To overcome this limited freedom of movements within the environment, 3D models of some buildings have been developed.

Many costly technologies, such as 3D scanning methods, are available for 3D digitization of cultural heritage artifact [16]. However, depending on the goal of the modeling, low-cost solutions hardware and software have been developed. 

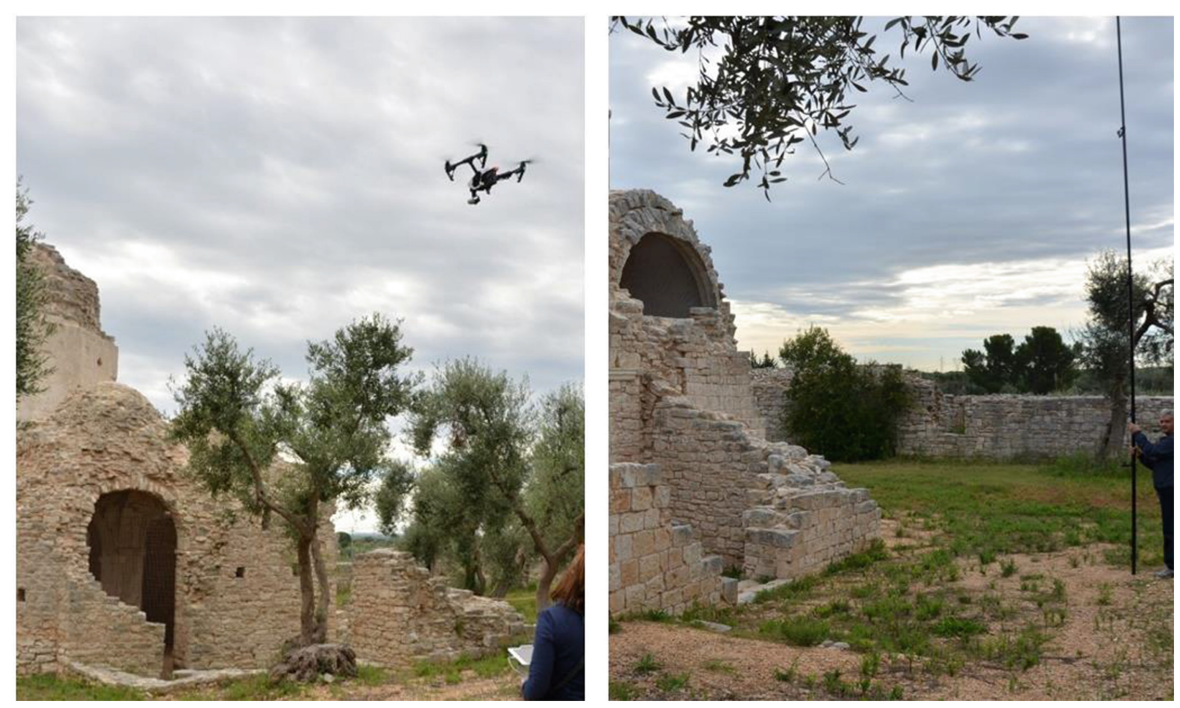

Fig. 8. The 3D model was obtained using SfM software by means of images by a camera mounted on an unmanned aerial vehicle (left) and by a multi-camera system ad hoc-developed (right).

The innovative 3D modeling procedure presented here was used to build the two churches model using a 'structure from motion' (SfM) software by means of images taken by the following cameras:

- Zenmuse X3 (CMOS sensor 12.4Mp; f/2.8; FOV $20 \mathrm{~mm}$ ) integrated on an unmanned aerial vehicle 'Inspire 1' (Fig. 8, left) for the survey of remote objects (e.g., vaults and capitals) where physical access is delicate or impossible according [17];

- GoPro Hero3 + Black Edition (CMOS sensor 12Mp; f/2.8; FOV Ultrawide $17.2 \mathrm{~mm}$, medium $21.9 \mathrm{~mm}$, narrow $34.4 \mathrm{~mm}$ ) mounted on a multi-camera system ad hoc-developed (Fig. 8, right) operated from the ground, to cover the entire targeted object.

The Multi-camera System. In this work, six camera were used, arranged on a carbon fiber rod in different height positions. The procedure provides for establishing a path along which to position the system every meter, so assuring a superimposition of $50 \%$, acquiring synchronized images by each camera using a remote control.

To simplify the post-processing, the images acquired at each station point will be renamed according to whether it is an architectural survey around a monument (socalled 'convergent axis'), or a survey of a single facade (so-called 'parallel axis').

In the first case, the last photo of the first station must be followed by the first photo of the second station and so on forming a spiral path (Fig. 9, left).

In the second case, the last photo of the first station must be followed by the last photo of the second station and, proceeding backwards, resume again with the first photo of the next station, forming a serpentine path (Fig. 9, right). 

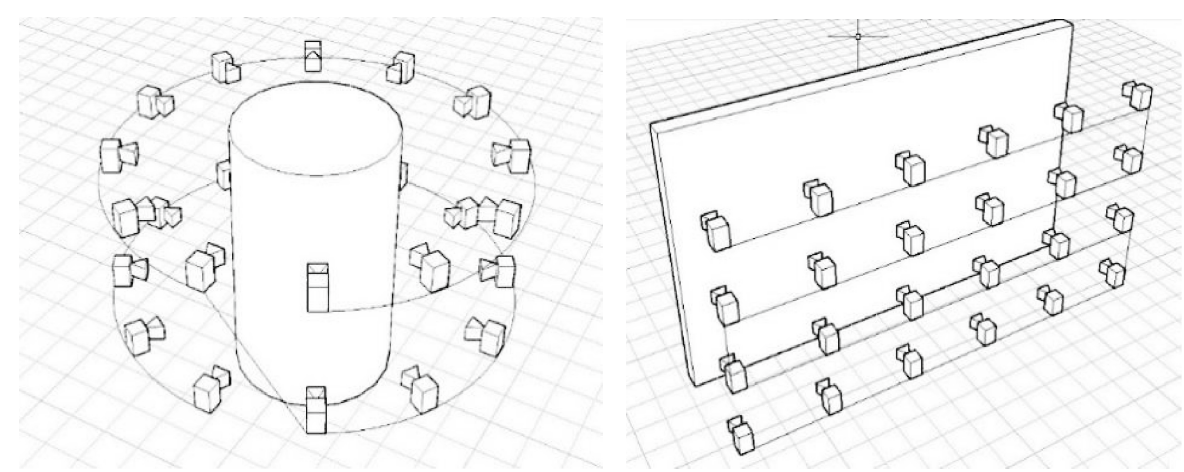

Fig. 9. Survey scheme with convergent axis (left), and parallel axis (right).

It should be emphasized that the sensor of the GoPro camera does not reflect the image quality standard required for a successful alignment process. This low quality is determined by the fisheye lens having a focal distance of $2.8 \mathrm{~mm}$ (equivalent in the camera reflex version to a $15.0 \mathrm{~mm}$ and therefore to a shooting range of $170^{\circ}$ ), installed on these cameras to shoot especially sports actions.

This wide-angle lens, despite having an excellent depth of field, has strong aberrations in the peripheral parts, prejudicing the process of recognizing homologous points. To reduce this problem, the frame of each camera is settled, through an internal menu, to the 'medium' value, so recording only the central part of the shot having reduced distortions. This operation, called 'cropping', reduces the final resolution to $7 \mathrm{Mp}$.

It should also be noted that the sensor on board these cameras belong to the range of economic sensors since each pixel has a size of just over $1.5 \mu$, far from the minimum $3 \mu$ recommended for obtaining a good image. It is true that remaining below this threshold, due to the increase in electronic circuitry and the amplification of the signal applied to each pixel; as a result, a background noise disturbing the image quality is generated. For this reason, it is necessary to determine in advance the Ground Sample Distance (GSD) value corresponding to how much real surface is captured in each pixel.

GSD $=$ pixel size $\times$ average shooting distance $/$ focal length $=1.7 \mathrm{~mm}$, where:

- the pixel size: $0.00154 \mathrm{~mm}$ (sensor width divided by the number of sensor pixels: $6.16 \mathrm{~mm} / 4000)$;

- the lens focal length: $2.77 \mathrm{~mm}$

- the average shooting distance: $3000 \mathrm{~mm}$

The GSD value obtained at an average distance of 3 meters, even if acceptable, was slightly higher than the recommended value, which should usually not exceed $1.5 \mathrm{~mm}$.

A solution to reduce the GSD value is to reduce the shooting distance, even if this involves a greater number of shots to preserve the overlap of $50 \%$ of the frames.

We made also a laser scanner survey to compare output and procedures, following the method already used [18]: the multi-camera is cheaper, requests basic skills and a reduced survey time; unfortunately processing time is very high using standard 
Table 1. Scanner laser vs Multi-camera system

\begin{tabular}{l|l|l}
\hline & Scanner laser & Multi-camera system \\
\hline Equipment cost (Euro) & 110,000 & 3,000 \\
\hline Total equipment weight (Kg) & 60 & 2 \\
\hline Parts to be assembled & 7 & 0 \\
\hline Acquisition process difficulty & Moderate & Low \\
\hline Stations performed & 25 & 250 \\
\hline Preparation times for each shot (minutes) & 15 & 0 \\
\hline Acquisition time for each shot (minutes) & 10 & 0.2 \\
\hline Total acquisition time (hours) & 10 & 1.5 \\
\hline Shot hight (meter) & Up to 1.5 & Up to 10 \\
\hline Data (GB) & 34 & 9 \\
\hline Data management time (hours) & 0 & 2 \\
\hline Processing time (days) & 1 & 15 \\
\hline Software cost (euro) & 10,000 & 3,000 \\
\hline
\end{tabular}

workstation; on the other hand, using a laser scanner only at ground level, gross errors occur for covers and recessed area (Table 1).

The 3D model produced appears as a faithful reproduction of the scene respect to the laser scan cloud of colored dots (supplementary image acquisition and software are needed to transform it into a mesh).

To post-process the images, two SfM 3D reconstruction software were used: Photoscan Agisoft and Zephyr 3DFlow. In both software, while performing the selfcalibration of the frames, their alignment did not give acceptable results.

Then we proceeded to calibrate the optics of the cameras using an application supplied with Photoscan (Agisoft Lens) that, by performing a thorough analysis on several shots to a grid pattern, generates a calibration file to be applied before alignment.

The 3D model obtained was then exported in 3DHop software, to be displayed in real-time and manipulated interactively (Fig. 10).

\subsection{Dissemination}

The goal was to find out how to develop an application accessible and able to combine all different types of data and output produced.

The dissemination tool is a multiplatform web site - described in the next paragraph - where users are interact with virtual tours and 3D models, with the support of text and sounds. Regardless of the order in which rooms are accessed, visitors are free to move around each environment, navigate the space and interact with it according to their own interests.

"You can't replicate that in VR, but you can give people a preview and understanding of what they would experience if they went to visit physically" says Abi Mandelbaum [19], CEO and co-founder of YouVisit (http://www.youvisit.com/), an organization that specializes in VR tours and works with more than 800 clients to drive 


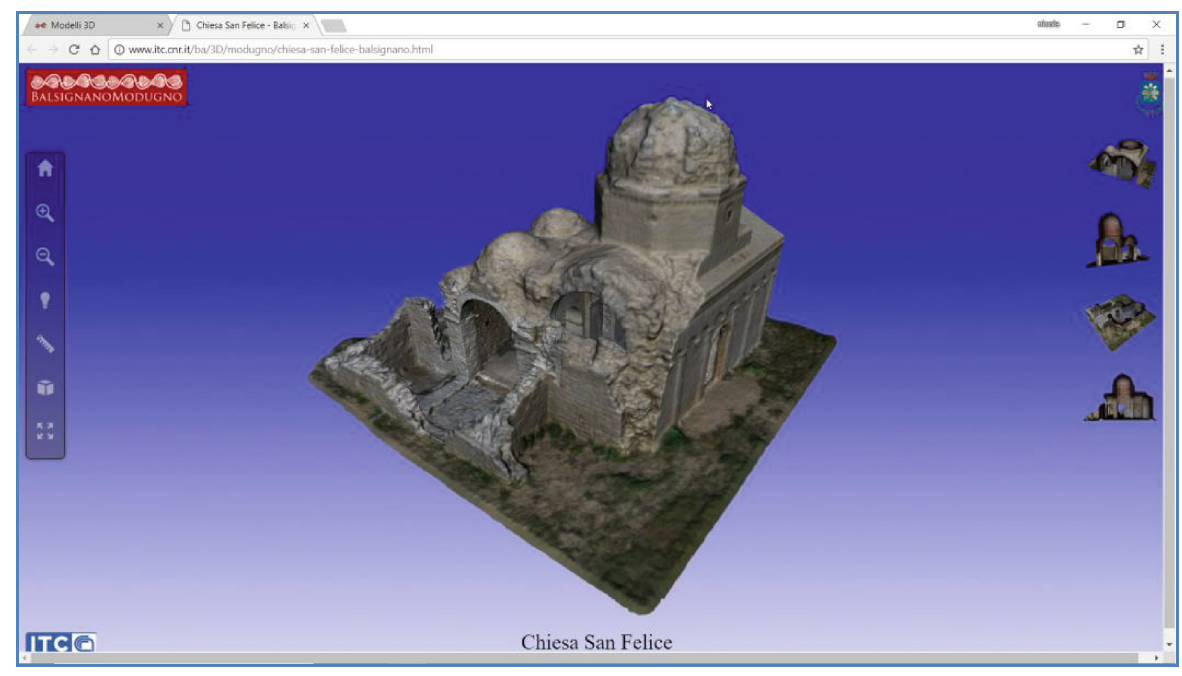

Fig. 10. 3D model displayed in real-time and manipulated interactively (3DHop software).

business results and engage consumers with interactive $360^{\circ}$ experiences. The University of Massachusetts Amherst published a study on the effects the VR has on people and travel destinations. The research showed that "VR generates pleasant emotions towards the destination even though it's a new form of advertisement" and VR heightens the sense of presence by determining people's interest, since they can engage much more than they could just by reading a simple tour guide; pointing out that people were more likely to share their VR experience on social media and talk to their friends about it $[20,21]$.

\section{The Web Site Implementation}

'When designing a web site layout there are some common mistakes that often appear' $[22,23]$. These mistakes cover not only design aspects but also general workflow tips that will get the job done nicely. We have supported the Municipality from concept through to post-implementation support of the web site; our goal was to not only provide a web solution but also to define features concretely, determine technology integration specifics, and prioritize anticipated future uses of the website to ensure that the system design is a scalable one.

The development process started with initial phase, 'ideation' [24]. During this phase, we worked with the Municipality to define the requirements for specific site functionality such as Virtual tours, 3D model, news, videos, social bookmarking, search, mobile device accessibility, and other such features. Designing a good website that combined strategic thinking and emerging technologies was a tricky balancing act to pull off. 
In this step, the team needed to work with all stakeholders to gather as much information as possible about how they believed the web- site should be function. At first, the Municipality did not have a web hosting service that allowed us to make initial website accessible via Internet and seen by anyone in real-time, by facilitating cooperation between stakeholders. In this regard, we decided to adopt a platform to build the website (site builder) and make it accessible via Internet by authorized users.

On the wide spectrum of website building solutions, Wix was the right choice and it has allowed us to build an initial model of the website. This platform is beginnerfriendly, has sleek designs, and boasts the easy drag and drop feature. Wix has become incredibly successful and has attracted over 103 million users to date; it has an extensive theme collection and many customization tools. Wix, with its users predesigned theme (more than 500) [25], has allowed us to give an idea on how to arrange website content and to share the web-solution with the Municipality.

At a later stage, the aim was to have a clear understanding of what the layout needs would be when designing sections beyond the homepage. Identifying the goals for the website, the functionality needed and the skills and resources to manage it helped us determine the right kind of web design for needs. The cost associated with managed services of a web site (to make functional changes, structural/layout changes, design changes, content changes, monitoring, and backups) is hard for Public Administration to estimate, plan and maintain. In addition, if we add that, in the Italian public administration, it is very difficult to find someone with experience in software development or website building [26]. The cost associated rises: 'According to the DESI (Digital Economy and Society Index) in 2016 in Italy $48 \%$ of the workforce has insufficient digital skills (EU average $37 \%$ ) on the basis of a $21 \%$ that has no digital competence and has never used the Internet (EU average 37\%)' [27].

The web-design step goes through those problems that cannot be resolved through simply solution but rather through a good layout, a clear hierarchy and a content easily navigable. The first thing to do is to come up with a top-level framework that solves all the design problems. This framework is the User Interface (UI) that surrounds the content and helps the user perform actions and navigate through it. UI includes the navigation and components like sidebars and bottom bars.

Our goal was to build a site that could work not only in the ideal scenario, but also in the worst-case scenario. For instance, a user could be using a small screen and check the site when there is barely any content on it so it looks broken. In this phase, we have focused on the need to emphasize usability, but also provide an attractive web presence for the Municipality. 'Current best practices call for spending about $10 \%$ of a design project's budget on usability' [28].

In many contexts, such as the tourist domain, creating a website version for each resolution and new device would be impossible or at least impractical. After gathering, these needs we have chosen a Content Management System (CMS) platform, a web site authoring and administration tools that could provide a reliable, secure, and costeffective content management solution to work more wisely and efficiently.

Therefore, even if Wix is designed and built specifically so it is easy for nondevelopers to use with ease, it is not an open source platform so its codes are not available to modify and may be some indirect costs involved, such as paying for external support. Today, free open source platforms to build a website are: 
- Joomla, the biggest CMS platform, mostly used;

- Drupal, a great CMS;

- WordPress, the perfect choice for small content oriented websites.

One of the main advantages of CMS is that it enables non-technically minded users to create functional pages or upload and modify content themselves, without having to outsource the work to a webmaster, or understand programming languages such as HTML5 or PHP.

The team has developed the final website using Joomla, a flexible, reliable, mobileready and user-friendly platform.

Unlike WordPress, which is mainly a blogging and small website platform, and unlike Drupal which is meant mostly for big websites. In the field of web design and development, we are quickly getting to the point of being unable to keep up with the endless new resolutions and devices.

To make the most of the Joomla benefits, we have defined according to [29]:

- Technical requirements of website;

- Workflows, processes and procedures to creating, publishing and managing digital content of website;

- Who should have access to the CMS, and at what level.

During the templating, programming and customization phases, we have adapted the base technologies selected in the design phase; we have merged the graphical design with the functionality by creating a graceful template, including the custom programming (modules or components) with the chosen Joomla template.

In addition, we have implemented the 'JA Image Hotspot' module [30], allowing to integrate into the website a responsive map (Fig. 11). It is a flexible extension used for page rendering to obtain an interactive map from a static image, filling in information for a specific area and adding markers with title and description. The module resizes the web map automatically based on the width of the user's browser window, preventing the pixel references within the image map from pushing some hotspots off of the screen.

The software solution consists of a mix of flexible grids and layouts, images and an intelligent use of CSS media queries. As the user switches from their laptop to iPad, the website automatically switches to accommodate for resolution, image size and scripting abilities.

This advanced solution eliminates the need for a different design and development phase for each user device. In this regard, we have integrated a 'Responsive Joomla Template' into the website.

With Responsive Templates the necessity to swipe around to find hidden sidebars, zooming in and out to make the text comfortable enough to read simply falls off: it fit all possible devices that have an access to the web. After all, the look and feel of mobile version reflects the original website. 


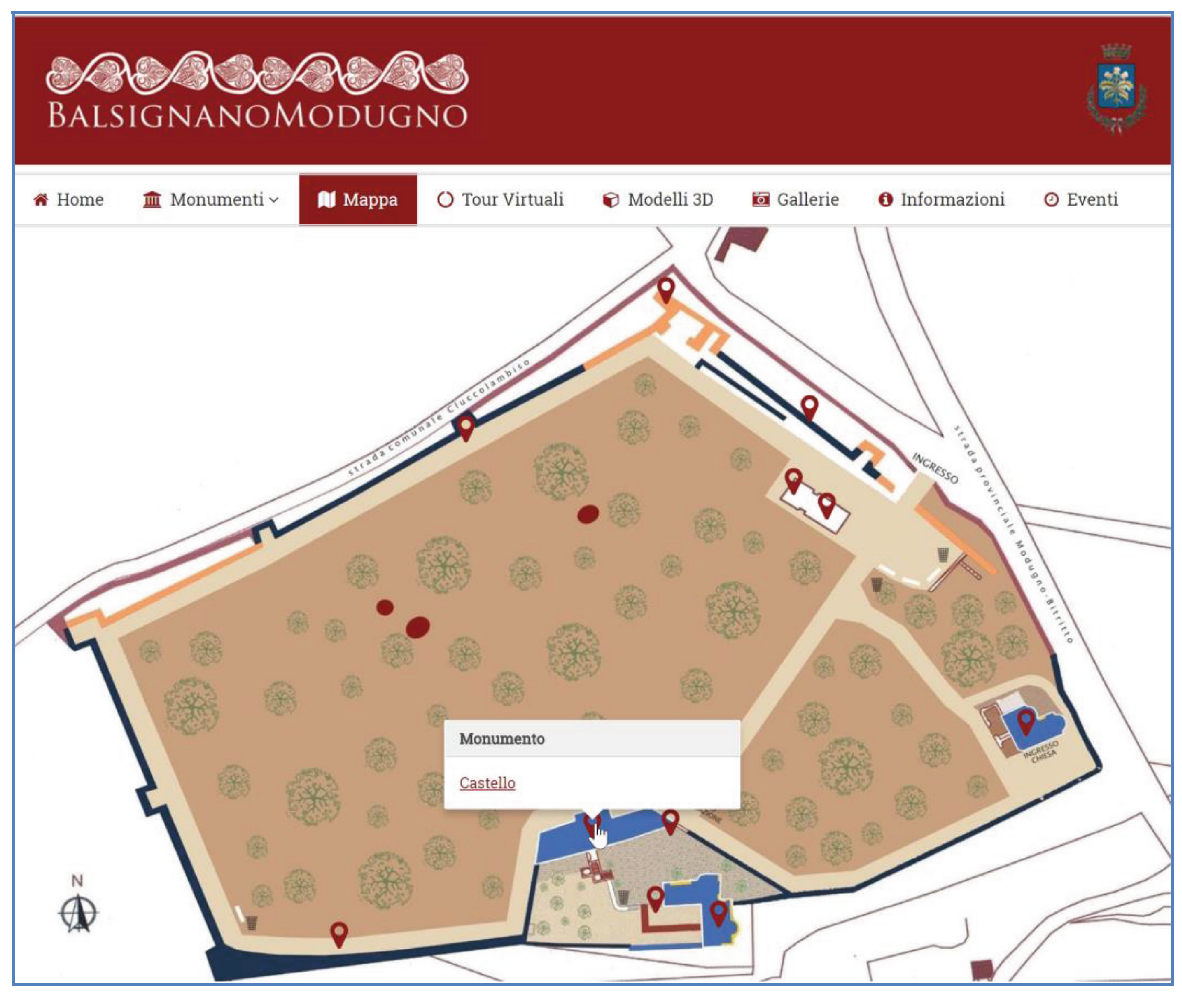

Fig. 11. The responsive web map in the website. The user can choose to display a tooltip (popover) when he clicks on the hotspot's position.

\section{Conclusions}

In our work, we developed an application that allows users to navigate the virtual representations and access the cultural resources in a dynamic way, according to their preferences and interests, thus experiencing 'Balsignano village' in an entertaining manner.

The application of digital techniques for the recording and visualizing the site of the 'Balsignano village' demonstrated how these tools can provide relevant and accurate information regarding the site's conservation as well as a posterity record in case of damage or further deterioration.

The proposed approach-interactive virtual tour and 3D models generated by images and as a whole constitutes an economic and practical alternative to the $3 \mathrm{D}$ scanner technology. Such integrated information can be beneficial both for accurate documentation and tourism.

It could be interesting to evaluate to what extent such an application can affect people's perceptions about 'Balsignano Village' and influence the will of actually visiting the place. Result of this evaluation could be useful to understand the power of virtual tours and $3 \mathrm{~d}$ models on current tourism. 
We have started to test 3D scenes reconstruction workflow using omni-directional camera Ricoh Teta Plus: it dramatically improves the performance of SfM software since the tracked interest points will not miss with a long base-line and sharp rotation [31].

Acknowledgments. This research was supported by the Municipality of Modugno (Italy) within the project 'Mu.S.A. - Must See Advisor'. We thank our colleagues from ITC-CNR who provided survey and outputs: Milella, N., Zonno, M., Battista, V., and Leandro, V.

Author Contributions. Maiellaro, N.: website project, testing, and 360 degree camera trials (Sects. 1, 2, 3); Varasano, A.: system architecture, and software development (Sect. 4); Capotorto, S.: 3D models (in Sect. 3).

\section{References}

1. Letellier, R.: Recording, documentation, and information management for the conservation of heritage places: guiding principles. J. Paul Getty Trust, Los Angeles (2007). https://www. getty.edu/conservation/publications_resources/pdf_publications/pdf/guiding_principles.pdf. Accessed 4 Apr 2018

2. Percy, K., Ward, S., Santana Quintero, M., Morrison, T.: Integrated digital technologies for the architectural rehabilitation \& conservation of Beinn Bhreagh Hall \& Surrounding Site, Nova Scotia, Canada. In: ISPRS Annals of the Photogrammetry, Remote Sensing and Spatial Information Sciences, vol. II-5/W3, pp. 235-241 (2015). https://www.isprs-annphotogramm-remote-sens-spatial-inf-sci.net/II-5-W3/235/2015/isprsannals-II-5-W3-2352015.pdf. Accessed 4 Apr 2018

3. Napolitano, R.K., Scherer, G., Glisic, B.: Virtual tours and informational modeling for conservation of cultural heritage sites. J. Cult. Herit. 29 (2017)

4. Bastanlar, Y., Grammalidis, N., Zabulis, X., Yilmaz, E., Yardimci, Y., Triantafyllidis, G.: 3D reconstruction for a cultural heritage virtual tour system. In: The International Archives of the Photogrammetry, Remote Sensing and Spatial Information Sciences, Beijing, vol. XXXVII, part B5, pp. 1023-1028 (2008). http://www.isprs.org/proceedings/XXXVII/ congress/5_pdf/177.pdf. Accessed 4 Apr 2018

5. Koeva, M., Luleva, M., Maldjanski, P.: Integrating spherical panoramas and maps for visualization of cultural heritage objects using virtual reality technology. Sensors 17, 829 (2017). http://www.mdpi.com/1424-8220/17/4/829/pdf. Accessed 4 Apr 2018

6. Freeman, M.A.: Not for casual readers: an evaluation of digital data from virginia archaeological websites. Master's thesis, University of Tennessee (2015). http://trace. tennessee.edu/cgi/viewcontent.cgi?article $=4785 \&$ context=utk_gradthes

7. The Colonial Williamsburg Foundation, eWilliamsburg. http://research.history.org/ ewilliamsburg/map.cfmTravel and Tourism Competitiveness Report (2015). http://www3. weforum.org/docs/TT15/WEF_Global_Travel\&Tourism_Report_2015.pdf

8. Maiellaro, N., Varasano, A.: BalsignanoModugno (2017). http://www.casaledibalsignano.it. Accessed 4 Apr 2018

9. Macina, R.: Balsignano - dal degrado al recupero. Edizioni Nuovi Orientamenti (2012). http://www.pugliadigitallibrary.it/media/00/00/74/957.pdf. Accessed 4 Apr 2018

10. Addison, A.C.: Emerging trends in virtual heritage. IEEE Multimed. 7, $22-25$ (2000) 
11. Balsignano, un insediamento rurale fortificato - Guida alla visita. Caggianelli, R. (ed.) Mario Adda Editore (2015)

12. Balsignano, un insediamento rurale fortificato - Archeologia, studi e restauri. Depalo, M.R., Pellegrino, E., Triggiani, M. (eds.) Mario Adda Editore (2015)

13. Fondo Simone. http://www.pinacotecabari.it/index.php/patrimonio-museale/patrimoniofotografico/fondo-simone. Accessed 4 Apr 2018

14. Eiris, R., Moud, H.I., Gheisari, M.: Using 360-degree interactive panoramas to develop virtual representations of construction sites. In: LC3 2017: Volume I - Proceedings of the Joint Conference on Computing in Construction (JC3), 4-7 July 2017, Heraklion, Greece, pp. 775-782 (2017)

15. Popovic, V., Seyid, K., Cogal, Ö., Akin, A., Leblebici, Y.: Design and Implementation of Real-Time Multi-Sensor Vision Systems. Springer, Cham (2017). https://doi.org/10.1007/ 978-3-319-59057-8

16. Santos, P., et al.: CultLab3D — on the verge of 3D mass digitization. In: Proceedings of the Eurographics Workshop on Graphics and Cultural Heritage, The Eurographics Association, Aire-la-Ville, Switzerland, 6-8 October 2014, pp. 65-74 (2014)

17. Louiset, T., Pamart, A., Gattet, E., Raharijaona, T., De Luca, L., Ruffier, F.: A shapeadjusted tridimensional reconstruction of cultural heritage artifacts using a miniature quadrotor. Remote Sens. 8, 858 (2016)

18. Maiellaro, N., Zonno, M., Lavalle, P.: Laser scanner and camera-equipped UAV architectural surveys. In: The International Archives of the Photogrammetry, Remote Sensing and Spatial Information Sciences, Volume XL-5/W4, 2015 3D Virtual Reconstruction and Visualization of Complex Architectures, 25-27 February 2015, Avila, Spain, pp. 381-386 (2015)

19. Mandelbaum, A.: Immersive Technology Expert. Co-Founder \& CEO of YouVisit. https:// www.linkedin.com/in/abimandelbaum. Accessed 13 May 2018

20. Griffin, T., Giberson, J., Lee, S.H.M., Guttentag, D., Kandaurova, M.: Virtual reality and implications for destination marketing. In: TTRA International Conference (2017). https:// scholarworks.umass.edu/cgi/viewcontent.cgi?referer $=\&$ httpsredir $=1 \&$ article $=$ 2103\&context=ttra. Accessed 13 May 2018

21. Tussyadiah, L., Wang, D., Jia, C.H.: Exploring the persuasive power of virtual reality imagery for destination marketing. In: TTRA International Conference (2016). https:// scholarworks.umass.edu/cgi/viewcontent.cgi?referer $=\&$ httpsredir $=1 \&$ article $=$ 1180\&context=ttra. Accessed 13 May 2018

22. Guglieri, C.: 23 steps to the perfect website layout (2017). https://www.creativebloq.com/ web-design/steps-perfect-website-layout-812625. Accessed 4 Apr 2018

23. Spolan, S.: Common website design problems (2016). https://www.zivtech.com/blog/8common-website-design-problems. Accessed 4 Apr 2018

24. Dam, R., Teo, S.: What is ideation - and how to prepare for ideation sessions (2018). https:// www.interaction-design.org/literature/article/what-is-ideation-and-how-to-prepare-forideation-sessions. Accessed 4 Apr 2018

25. Wix Review 2017 - The Main Pros \& Cons. Wix advantages and disadvantages (2017). http://www.wittythemes.com/wix-review. Accessed 4 Apr 2018

26. Rapari, G.: Una PA senza competenze digitali, Assintel: 'Prenda esempio dalle aziende' (2017). https://www.agendadigitale.eu/cultura-digitale/una-pa-senza-competenze-digitaliassintel-prenda-esempio-dalle-aziende. Accessed 4 Apr 2018

27. Carlizzi, D.N., Quattrone, A., D’Errigo, F.: Sblocco del turn-over: occasione per introdurre futuro nelle PA (2017). https://www.agendadigitale.eu/cultura-digitale/una-pa-senzacompetenze-digitali-assintel-prenda-esempio-dalle-aziende. Accessed 4 Apr 2018 
28. Nielsen, J.: Usability 101: Introduction to Usability (2012). https://www.nngroup.com/ articles/usability-101-introduction-to-usability. Accessed 4 Apr 2018

29. Content management systems - Advantages of using a content management system. https:// www.nibusinessinfo.co.uk/content/advantages-using-content-management-system. Accessed 4 Apr 2018

30. JA Image Hotspot (2018). https://www.joomlart.com/joomla/extensions/ja-image-hotspotmodule. Accessed 4 Apr 2018

31. Song, M., Watanabe, H., Hara, J.: Robust 3D reconstruction with omni-directional camera based on structure from motion. In: Proceedings of the International Workshop on Advanced Image Technology 2018, 7-9 January 2018, Chiang Mai, Thailand (2018). http://www. iwait2018.org/Paper\%20IWAIT2018/IWAIT2018_paper_105.pdf. Accessed 4 Apr 2018

Open Access This chapter is licensed under the terms of the Creative Commons Attribution 4.0 International License (http://creativecommons.org/licenses/by/4.0/), which permits use, sharing, adaptation, distribution and reproduction in any medium or format, as long as you give appropriate credit to the original author(s) and the source, provide a link to the Creative Commons licence and indicate if changes were made.

The images or other third party material in this chapter are included in the chapter's Creative Commons licence, unless indicated otherwise in a credit line to the material. If material is not included in the chapter's Creative Commons licence and your intended use is not permitted by statutory regulation or exceeds the permitted use, you will need to obtain permission directly from the copyright holder. 